\title{
16
}

\section{An Improved Search Strategy for Lossy Channel Systems}

\author{
Parosh Aziz Abdulla \\ Uppsala University, Dept. of Computer Systems \\ P.O. Box 325, S-751 05 Uppsala, Sweden, paroshedocs . uu . se. \\ Mats Kindahl \\ Uppsala University, Dept. of Computer Systems \\ P.O. Box 325, S-751 05 Uppsala, Sweden, parosh@docs . uu . se.
}

\author{
Doron Peled \\ Bell Laboratories \\ 700 Mountain Avenue, Murray Hill, NJ 07974, \\ doroneresearch.bell-labs.com.
}

\begin{abstract}
In [1] we considered lossy channel systems which are a particular class of infinite state systems consisting of finite state processes communicating through channels that are unbounded and unreliable. We presented a backward reachability algorithm which, starting from a set of "bad" states, checks whether there is a backward path to the initial state of the system. Using standard techniques, the reachability algorithm can be used to check safety properties for lossy channel systems.

In this paper we adopt partial order techniques to improve the algorithm in [1]. We define a preorder, which we call the better than relation, among the set of transitions of the system. Intuitively a transition is better than another if choosing the first transition instead of the second preserves the reachability of the initial state during the analysis. This relation is weaker than the independence relation, which is an equivalence relation, used in traditional partial order methods, in the sense that two transitions are independent if and only if each of them is better than the other. Consequently, our method gives a better reduction in the number of states considered during the analysis. We demonstrate the efficiency of the approach by a number of experimental results.
\end{abstract}

\section{INTRODUCTION}

In the last few years, there has been a considerable interest in algorithmic verification of distributed and parallel systems. The research has led to the discovery of numerous efficient methods for the verification of finite-state systems ([7], [9], [14], [20], etc.). An obvious limitation of these methods is that systems with infinitely many states fall beyond their capabilities. Recently, algorithmic verification methods have been developed for some classes of infinite-state systems.

A particular class of infinite-state systems which has been important in the analy- 
sis of, e.g. , communication protocols consists of finite-state processes that communicate via unbounded FIFO channels $[6,5]$. Such systems are infinite-state due to the unboundedness of the channels, and it is well-known that these systems can simulate Turing machines, and hence most interesting verification problems are undecidable for them [6]. In an earlier work [1], we considered a variant of this class, called lossy channel systems, where the FIFO channels are unreliable, in the sense that they may nondeterministically lose messages. In spite of this restriction, lossy channel systems can be used to model and analyze many interesting systems, e.g. link protocols such as the Alternating Bit Protocol [4] and HDLC [15], which are designed to operate correctly even in the case that the FIFO channels are faulty and may lose messages.

In $[1,2]$ we present a reachability algorithm for lossy channel systems which, for any state, checks whether the state is reachable from the initial state of the system. The idea of the algorithm is to define a partial order $\preceq$ on the set of states, where one state is smaller than another if the two states differ only in that the content of each channel in the first state is a (not necessarily contiguous) substring of the content of the same channel in the second state. An important property of lossy channel systems is that their behaviour is monotone with respect to $\preceq$, i.e. larger states have transitions to larger states. The algorithm operates on ideals, where the ideal generated by a state is the set of states larger than or equal to that state. The reachability of a state $\gamma$ is obviously equivalent to the reachability of the ideal generated by $\gamma$, since if there is a path from the initial state to any state $\gamma^{\prime}$, which is larger than $\gamma$, then we can continue from $\gamma^{\prime}$ losing messages until we obtain $\gamma$. To check the reachability of an ideal $I$, we perform a reachability analysis starting from $I$ and going backwards. At each step we pick an ideal $I^{\prime}$ which has already been generated during the analysis, and compute the set $\operatorname{pre}(I)$ of states from which $I^{\prime}$ is reachable through the application of a single step of the transition relation. It is shown in $[1,2]$ that the monotonicity of lossy channel systems implies that $\operatorname{pre}(I)$ is also an ideal and that it is in fact computable.

Partial order reductions $[18,12,19]$ are a family of techniques which can be used to perform more efficient verification of systems consisting of asynchronously communicating concurrent processes, e.g. lossy channel systems. These methods are based on the observation that concurrent actions of the processes are often independent and hence their interleavings are equivalent in that they all lead to the same states. Most existing partial order methods work with a forward search of the state space, starting from the initial state and trying to find a path forwards to a final state. Action sequences are grouped into equivalence classes, for each of which at least one representative must to be analyzed. The algorithm then searches a reduced state-space, where at each step only a subset (an ample set [18]) of the enabled transitions is considered. The requirement is that an ample set should contain at least one representative of each equivalence class.

In this paper, we employ ideas based on partial order reductions to derive a more efficient version of the algorithm in [1,2]. Instead of working with an equivalence relation (the independence relation), we define a preorder (the "better than" relation) among actions. An event $\alpha$ is better than an event $\beta$ in a state $\gamma$ if $\alpha$ followed by $\beta$ leads to a smaller state (with respect to $\preceq$ ) from $\gamma$ than $\beta$ followed by $\alpha$. We extend the preorder to sequences of actions, where a sequence is "better than" another if the first sequence can be obtained from the second by permuting adjacent actions so that "better" actions occur earlier in the first sequence than in the second sequence. We 
show that it is sufficient, for each set of sequences, to consider only the "best" elements of the set. The correctness of our approach can be shown again to follow from monotonicity of lossy channel systems.

Our method differs from traditional partial order techniques in the following aspects.

- We work with a preorder (the "better than" relation) which is weaker than the independence relation, since two sequences are equivalent if and only if each of them is better than the other. Instead of choosing representatives of each equivalent class, it is sufficient to choose the best elements of a certain set of sequences. This leads to a more efficient search algorithm, since two sequences may not be equivalent, but one of them may still be discarded from the analysis, if it is "worse" than the other.

- We apply our methods in the context of backward reachability analysis. This is necessary since the reachability algorithm for lossy channel systems operates backwards. In fact it is shown in [10] that forward reachability analysis is infeasible for lossy channel systems.

- The reachability algorithm works on ideals, so our method can be considered to operate on sets of states (ideals) rather than individual states. Since ideals are infinite sets, each ideal is represented symbolically by its set of generators. This set of generators can be shown always to be finite.

Using standard techniques [20], we can check safety properties for lossy channel systems through a reduction to the reachability problem.

In fact our method is quite general. In $[10,3]$ general theories are presented for the verification of systems with monotone behaviour. Examples of such systems include besides lossy channel systems, Petri nets, real-time automata, relational automata, Basic Parallel Processes (BPPs), and certain classes of parametrized systems. The general reachability algorithm presented in [3] exploits the monotonicity of the system in the same manner as the algorithm for lossy channel systems. Consequently our techniques are applicable even for these classes of systems.

In the next section we introduce lossy channel systems and some properties of these. In section 3 we introduce the reachability algorithm. In section 4 we introduce the better-than relation and some properties of action sequences. In section 5 we show the modifications needed to improve the reachability algorithm. In section 6 we give an algorithm to compute ample sets. In section 7 we show some experimental results. In section 8 we give some conclusions and directions for future research.

\section{PRELIMINARIES}

A lossy channel system consists of a control part and a channel part. The control part is modelled as a number of finite-state processes communicating via the channels, while the channel part consists of a finite set of channels. Each channel behaves as a FIFO buffer which is unbounded and unreliable in the sense that it can lose messages. A channel is used to perform asynchronous communication between a pair of processes, so for each channel there is unique process sending messages to the channel, and a unique process receiving messages from the channel. 
For a tuple $\vec{x}=\left\langle x_{1}, x_{2}, \ldots, x_{n}\right\rangle$, we use $\vec{x}(i)$ to denote $x_{i}$ and $\vec{x}[i \mapsto e]$ to denote the tuple $\left\langle x_{1}, x_{2}, \ldots, x_{i-1}, e, x_{i+1}, \ldots, x_{n}\right\rangle$. The domain $\operatorname{dom}(\vec{x})$ of $\vec{x}$ is the set $\{1,2, \ldots, n\}$ of indices, while the range $\operatorname{rng}(\vec{x})$ of $\vec{x}$ is the set $\left\{x_{1}, x_{2}, \ldots, x_{n}\right\}$ of values. For strings $x$ and $y$, we use $x \cdot y$ to denote the concatenation of $x$ and $y$.

Formally, lossy channel systems are defined as follows.

Definition 1 A lossy channel system is a tuple $\mathcal{L}=\left\langle\vec{S}, \vec{s}_{0}, A, C, M, \delta\right\rangle$, where

$\vec{S}=S_{1} \times \cdots \times S_{n}$ is a tuple of finite sets of control states, where $n$ is the number of processes in the control part, and $S_{i}$ denotes the set of control states of the $i^{\text {th }}$ process.

$\vec{s}_{0} \in \vec{S}$ is a tuple of initial control states,

$A$ is a finite set of actions,

$C$ is a finite set of channels,

$M$ is a finite set of messages,

$\delta$ is a finite set of transitions, each of which is a triple of the form $\left\langle s_{1}^{i}, l, s_{2}^{i}\right\rangle$, where $s_{1}^{i}, s_{2}^{i} \in \vec{S}(i)$ for some process $i$, and $l$ is of the form

$c ! m$ where $c \in C$ and $m \in M$, representing sending a message $m$ to channel $c$ ( $m$ is appended to the end of $c$ );

$c ? m$ where $c \in C$ and $m \in M$, representing receiving a message $m$ from channel $c$ ( $m$ is removed from the head of $c$ ); or

$a$ where $a \in A$, representing an observable interaction with the environment without changing the contents of the channels.

A global state (or just state) $(\vec{s}, \vec{w})$ consists of a tuple $\vec{s} \in \vec{S}$ of control states and a tuple $\vec{w}$ of strings over $M$. The string $\vec{w}$ is indexed by the elements of $C$, with $\vec{w}(c)$ representing the content of $c$. We use $\gamma$ to denote a global state and use $\Gamma$ to denote the set of all global states.

We formalise the intuitive behaviour of lossy channel systems by introducing a labelled transition system on global states.

Definition 2 Given a lossy channel system $\mathcal{L}=\left\langle\vec{S}, \vec{s}_{0}, A, C, M, \delta\right\rangle$ we construct the labelled transition system $\mathcal{G}=\langle\Gamma, \longrightarrow, A \cup\{\tau\}\rangle$ where $\Gamma$ is the set of global states, $A \cup\{\tau\}$ is a set of labels, and $\longrightarrow \subseteq \Gamma \times(A \cup\{\tau\}) \times \Gamma$ is a transition relation among global states. We will use $\gamma_{1} \stackrel{a}{\longrightarrow} \gamma_{2}$ to denote $\left(\gamma_{1}, a, \gamma_{2}\right) \in \longrightarrow$. The set $\longrightarrow$ is the smallest set such that

- if $\left\langle s_{1}^{i}, c ! m, s_{2}^{i}\right\rangle \in \delta$ and $(\vec{s}, \vec{w})$ is a global state such that $\vec{s}(i)=s_{1}^{i}$, then $(\vec{s}, \vec{w}) \stackrel{\tau}{\longrightarrow}$ $\left(\vec{s}\left[i \mapsto s_{2}^{i}\right], \vec{w}[c \mapsto \vec{w}(c) \cdot m]\right)$. This corresponds to sending message $m$ to channel $c$.

- If $\left\langle s_{1}^{i}, c ? m, s_{2}^{i}\right\rangle \in \delta$ and $(\vec{s}, \vec{w})$ is a global state such that $\vec{s}(i)=s_{1}^{i}$ and $\vec{w}(c)=m$. $v$, then $(\vec{s}, \vec{w}) \stackrel{\tau}{\longrightarrow}\left(\vec{s}\left[i \mapsto s_{2}^{i}\right], \vec{w}[c \mapsto v]\right)$. This corresponds to receiving message 
$m$ from channel $c$. Notice that this transition may be performed only if the element in the head of channel $c$ is equal to $m$.

- If $\left\langle s_{1}^{i}, a, s_{2}^{i}\right\rangle \in \delta$ and $(\vec{s}, \vec{w})$ is a global state such that $\vec{s}(i)=s_{1}^{i}$, then $(\vec{s}, \vec{w}) \stackrel{a}{\longrightarrow}$ $\left(\vec{s}\left[i \mapsto s_{2}^{i}\right], \vec{w}\right)$. This corresponds to performing an observable interaction $a$ with the environment.

- If $(\vec{s}, \vec{w})$ is a global state such that $\vec{w}(c)=x \cdot m \cdot y$, then $(\vec{s}, \vec{w}) \stackrel{\tau}{\longrightarrow}(\vec{s}, \vec{w}[c \mapsto x \cdot y])$. This corresponds to losing message $m$ from channel $c$.

We use $\left(\vec{s}_{1}, \vec{w}_{1}\right) \longrightarrow\left(\vec{s}_{2}, \vec{w}_{2}\right)$ to denote that $\left(\vec{s}_{1}, \vec{w}_{1}\right) \stackrel{a}{\longrightarrow}\left(\vec{s}_{2}, \vec{w}_{2}\right)$ for some $a \in A \cup$ $\{\tau\}$, and use $\stackrel{*}{\longrightarrow}$ to denote the transitive closure of $\longrightarrow$.

We define a partial order $\preceq$ on elements of $M^{*}$ by letting $w_{1} \preceq w_{2}$ iff $w_{1}$ is a (not necessarily contiguous) substring of $w_{2}$, e.g. given $M=\{a, b, c, x, y, z\}, a b \preceq$ $a b c \preceq a x b y c \npreceq a x y c$. We use $\varepsilon$ to denote the empty string. We extend the partial order $\preceq$ to states by letting $\left(\vec{s}_{1}, \vec{w}_{1}\right) \preceq\left(\vec{s}_{2}, \vec{w}_{2}\right)$ iff $\vec{s}_{1}=\vec{s}_{2}$ and $\vec{w}_{1}(c) \preceq \vec{w}_{2}(c)$ for each $c \in C$. An interesting property of this partial order is that it is a well quasi-order, i.e. there is no infinite set of strings such that all strings are pairwise incomparable.

Lemma 1 (Higman's Lemma) Let $M$ be a finite set. If $S \subseteq M^{*}$ is a subset such that all strings in $S$ are pairwise incomparable with respect to $\preceq$, then $S$ is finite.

The proof of this lemma can be found in [17], where it is attributed to Higman [13].

The well quasi-orderedness of our partial order on global states follows directly from the lemma.

A set $I$ of states is said to be an ideal if it is the case that $\gamma \in I$ and $\gamma \preceq \gamma^{\prime}$ implies $\gamma^{\prime} \in I$. For a state $\gamma$, the ideal generated by $\gamma$ is the set $\left\{\gamma^{\prime} ; \gamma \preceq \gamma^{\prime}\right\}$.

\section{A REACHABILITY ALGORITHM}

In this section we describe a reachability algorithm $[1,2]$ for lossy channel systems.

The initial state of a lossy channel system (denoted $\iota$ ) is given by $\left(\vec{s}_{0}, \vec{w}_{0}\right)$, where $\vec{s}_{0}$ is the initial control state and $\vec{w}_{0}(c)=\varepsilon$ for each $c \in C$. We say that a state $\gamma$ is reachable if $\iota \stackrel{*}{\longrightarrow} \gamma$. We define the reachability problem formally as follows.

\section{Definition 3 [Reachability Problem]}

Instance: A lossy channel system $\mathcal{L}=\left\langle\vec{S}, \vec{s}_{0}, A, C, M, \delta\right\rangle$ and a set $F \subseteq \Gamma$ of final states.

Question: Is there a state $\gamma \in F$ such that $\left(\vec{s}_{0}, \vec{w}_{0}\right) \stackrel{*}{\longrightarrow} \gamma$.

The set $F$ is usually used to represent a set of "bad" states which we do not want to occur during the execution of the system. It can be shown $[1,2]$ that, using standard techniques [20], all safety properties, formulated as regular sets of allowed finite traces, can be reduced to the reachability problem.

To decide reachability, we perform a backward reachability analysis, starting with the states in $F$ and try to find a path back to $\iota$. Since the state space is infinite, the analysis is not a priori bounded. It turns out that it is inconvenient to choose the inverse 
of the forward transition relation (i.e. $\longrightarrow^{-1}$ ) as our basic backward transition step, since this would add messages to the channels in an uncontrolled manner. Instead we define a "backward" semantics for lossy channel systems as follows.

Let an operation be a partial function from states to states. An operation $\alpha$ is enabled at a state $\gamma$ if $\alpha(\gamma)$ is defined for $\gamma$. The set of all enabled operations at state $\gamma$ is denoted enabled $(\gamma)$.

Definition 4 Let $(\vec{s}, \vec{w})$ be a state of a lossy channel system. We define three different operations, each of which corresponds to going backwards in the transition relation. Notice that we execute the operations in reverse, e.g. receiving corresponds to adding a message to the beginning of a channel, etc.

rcv $c, m$ A backwards receive operation. Enabled from $(\vec{s}, \vec{w})$ if $\left\langle s_{2}^{i}, c ? m, s_{1}^{i}\right\rangle \in \delta$ and $\vec{s}(i)=s_{1}^{i}$. When executed, the state $\left(\vec{s}\left[i \mapsto s_{2}^{i}\right], \vec{w}[c \mapsto m \cdot \vec{w}(c)]\right)$ is obtained. snd $c, m$ A backwards send operation. Enabled from $(\vec{s}, \vec{w})$ if $\left\langle s_{2}^{i}, c ! m, s_{1}^{i}\right\rangle \in \delta$ and $\vec{s}(i)=s_{1}^{i}$. When executed, the state $\left(\vec{s}\left[i \mapsto s_{2}^{i}\right], \vec{w}[c \mapsto v]\right)$ is obtained if $\vec{w}(c)=$ $v \cdot m$ for some $v \in M^{*}$, otherwise $\left(\vec{s}\left[i \mapsto s_{2}^{i}\right], \vec{w}\right)$ is obtained, corresponding to a send with a subsequent message loss.

Icl A backwards local operation. Enabled from $(\vec{s}, \vec{w})$ if $\left\langle s_{2}^{i}, a, s_{1}^{i}\right\rangle \in \delta$ and $\vec{s}(i)=$ $s_{1}^{i}$. When executed, the state $\left(\vec{s}\left[i \mapsto s_{2}^{i}\right], \vec{w}\right)$ is obtained.

Intuitively, for a state $\gamma$ and an operation $\alpha$, if $I$ and $I^{\prime}$ are the ideals generated by $\gamma$ and $\alpha(\gamma)$ respectively, then $I^{\prime}$ is the set of states from which a state in $I$ is reachable through a single application of $\alpha$.

We are now ready to introduce the algorithm to decide reachability for lossy channel systems [2]. The algorithm uses a set $V$ of "visited" states, and a "working" set $W$ of states which are yet to be investigated. The algorithm proceeds by selecting and removing a state $\gamma$ from the working set $W$. From $\gamma$ the set of all enabled operations enabled $(\gamma)$ is computed. Each operation is used to compute a predecessor state $\alpha(\gamma)$, which is in turn added to the working set $W$. The state $\gamma$ is then added to the set $V$. Observe that, during our search, if we find a state larger than some state in $V$, we know that the state is redundant since it gives no new information, hence we can throw it away. A detailed description of the algorithm can be seen in Figure 1.

Theorem 2 The reachability algorithm is correct in the sense that it always terminates, and it returns the value true if and only if a state in $F$ is reachable.

The proof can be found in [2], where termination of the algorithm is shown to follow from Lemma 1.

\section{DEPENDENCY AMONG OPERATIONS}

In this section we define a dependency relation among operations.

For a state $\gamma$ and a sequence of operations $\rho=\alpha_{1} \alpha_{2} \cdots \alpha_{n}$, we say that $\rho$ is enabled from $\gamma$ if, for each $1 \leq i<n, \alpha_{i}$ is enabled from $\alpha_{i-1}\left(\alpha_{i-2}\left(\ldots \alpha_{2}\left(\alpha_{1}(\gamma)\right)\right)\right)$. If $\rho$ is enabled from $\gamma$ then we use $\rho(\gamma)$ to denote the state $\gamma^{\prime}=\alpha_{n}\left(\cdots \alpha_{2}\left(\alpha_{1}(\gamma)\right)\right)$. 

Algorithm 1 (Reachability Algorithm)
Input: A lossy channel system $\mathcal{L}$ and a set $F$ of states. states to be investigated.
1) Add all states in $F$ to the set $W$
2) If $W$ is empty, exit with the result false
3) Select and remove a state $\gamma \in W$
4) If $\gamma=\iota$, exit with the result true
5) If there is $\gamma^{\prime} \in V$ such that $\gamma^{\prime} \preceq \gamma$, goto 2
6) For each operation $\alpha \in \operatorname{enabled}(\gamma)$
7) Add $\alpha(\gamma)$ to $W$
8) Add $\gamma$ to $V$
9) Goto 2

Output: true if any state in $F$ is reachable, false otherwise.

Local: A set $V$ containing the already visited states and a set $W$ containing

Figure 1 Reachability Algorithm

An operation $\alpha$ is monotone iff $\gamma \preceq \gamma^{\prime}$ implies $\alpha(\gamma) \preceq \alpha\left(\gamma^{\prime}\right)$. Observe that this means that $\alpha$ is enabled in both $\gamma$ and $\gamma^{\prime}$.

Proposition 3 The operations snd $c, m, \operatorname{rcv} c, m$ and $\mathrm{lcl}$ are monotone.

Proof. Follows directly from the definitions.

For a state $\gamma$ and operations $\alpha$ and $\beta$, we say that $\alpha$ is better than $\beta$ in $\gamma$ when executing $\alpha$ before $\beta$ results in a smaller state with respect to $\preceq$. It is defined formally as follows.

Definition 5 An operation $\alpha$ is better than an operation $\beta$ in a state $\gamma$ (denoted $\beta \sqsubseteq_{b}$ $\alpha)$ if and only if:

- $\alpha, \beta \in \operatorname{enabled}(\gamma)$,

- $\alpha \in \operatorname{enabled}(\beta(\gamma))$,

- $\beta \in \operatorname{enabled}(\alpha(\gamma))$, and

- $\alpha \beta(\gamma) \preceq \beta \alpha(\gamma)$.

If $\beta \sqsubseteq_{b} \alpha$ and $\alpha \sqsubseteq_{b} \beta$ then $\alpha$ and $\beta$ are independent and if $\beta \Phi_{b} \alpha$ and $\alpha \square_{b} \beta$ the operations are dependent.

Since $\alpha \beta(\gamma) \preceq \beta \alpha(\gamma)$, it is "better", from the point of view of the reachability algorithm, to perform $\alpha$ followed by $\beta$ than the opposite. The reason is that we know that smaller states generate larger ideals. Consequently, it is safe to discard the sequence $\beta \alpha$ since the ideal generated by $\beta \alpha(\gamma)$ is a subset of the ideal generated by $\alpha \beta(\gamma)$.

Proposition 4 Given lossy channel system $\mathcal{L}$ with operations snd $c, m, \operatorname{rcv} c, m$, and Icl enabled in a state $(\vec{s}, \vec{w})$. 
1. Operations of the same process are always dependent.

2. $\operatorname{rcv} c, n$ is better than snd $d, m$ iff $c=d, \vec{w}(c)=\varepsilon$, and $m=n$; otherwise they are independent.

3. all other combinations are independent.

Proof. Follows directly from the definitions.

Notice that the operation snd $c, n$ has two possible behaviours, depending on the state from which it is taken. The fact that $\operatorname{rcv} c, n$ is better than snd $c, n$ makes use of this, replacing one type of execution by another.

Definition 6 Let $\gamma$ be a state, $\rho$ be a finite sequence such that $\rho \in \operatorname{enabled}(\gamma)$ and $\alpha$ an operation. An operation $\alpha$ is contributory to $\rho$ from state $\gamma$ iff for any partition of $\rho$ into $\sigma \beta \sigma^{\prime}$, where $\beta$ is an operation, $\beta \sqsubseteq_{b} \alpha$ holds in $\sigma(\gamma)$.

Intuitively, a contributory operation has the property that when it is moved forward in a sequence, we reach "smaller" states (according to $\preceq$ ).

Lemma 5 Let $\gamma$ be a state, $\rho$ be a finite sequence of monotone operations such that $\rho \in \operatorname{enabled}(\gamma)$, and $\alpha$ a single operation. If $\alpha$ is contributory to $\rho$ from $\gamma$ then $\alpha \rho(\gamma) \preceq \rho \alpha(\gamma)$.

Proof. Proof by induction on the length of $\rho$. For the base case $|\rho|=0$ the lemma holds trivially. For the induction case we let $\rho$ be a sequence of operations such that $|\rho|>0$ and $\alpha$ is contributory to $\rho$ from $\gamma$. Let $\rho=\rho^{\prime} \beta$ for some sequence of operations $\rho^{\prime}$ and some operation $\beta$. The operation $\alpha$ is better than every operation in $\rho$ and in particular the operation $\beta$. Hence $\alpha \beta \rho^{\prime}(\gamma) \preceq \beta \alpha \rho^{\prime}(\gamma)$, i.e.

$\rho^{\prime} \alpha \beta(\gamma) \preceq \rho^{\prime} \beta \alpha(\gamma)$

From the induction hypothesis we know that $\alpha \rho^{\prime}(\gamma) \preceq \rho^{\prime} \alpha(\gamma)$. Since $\beta$ is monotone and enabled at $\alpha \rho^{\prime}(\gamma)$ it follows that

$\alpha \rho^{\prime} \beta(\gamma) \preceq \rho^{\prime} \alpha \beta(\gamma)$

By combining (1) and (2) we see that $\alpha \rho^{\prime} \beta(\gamma) \preceq \rho^{\prime} \beta \alpha(\gamma)$.

In Lemma 5, we define a preorder $\sqsubseteq_{B}$ on sequences of operations, such that $\rho_{1} \sqsubseteq_{B}$ $\rho_{2}$ whenever $\rho_{1}=\rho^{\prime} \beta \alpha \rho^{\prime}$ and $\rho_{2}=\rho^{\prime} \alpha \beta \rho^{\prime}$, with $\beta \sqsubseteq_{b} \alpha$ in $\rho^{\prime}(\gamma)$. We define the "better than" relation among sequences to be the reflexive transitive closure $\complement_{B}^{*}$ of $\complement_{B}$. Intuitively a sequence $\rho_{2}$ is better than a sequence $\rho_{1}$, if $\rho_{2}$ can be obtained from $\rho_{1}$ by permuting adjacent actions so that "better" actions occur earlier in $\rho_{2}$ than in $\rho_{1}$. It follows from Lemma 5 that $\rho_{2}(\gamma) \preceq \rho_{1}(\gamma)$.

This approach is more general than that used in traditional partial order reduction methods, where $\complement_{b}$ is taken to be an equivalence relation and hence $\complement_{B}^{*}$ becomes an equivalence (rather than a preorder) on operation sequences. 

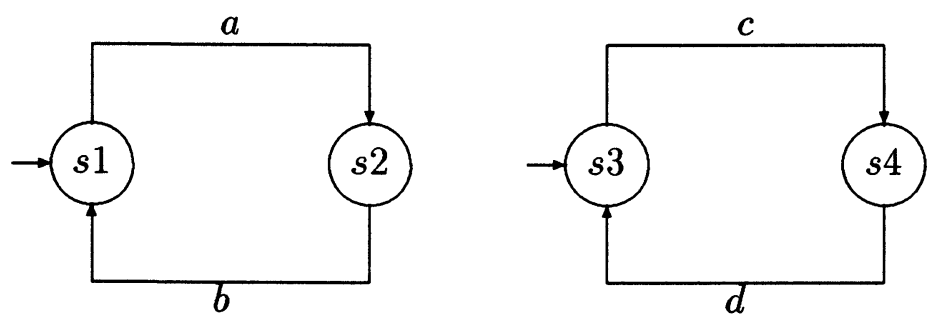

Figure 2 A system of two concurrent processes.

\section{IMPROVING THE REACHABILITY ALGORITHM}

In this section we introduce two strategies to improve the search conducted in Algorithm 1 . The general idea is to consider only a subset of the enabled operations for each state. We replace enabled $(\gamma)$ on line 6 in Algorithm 1 with ample $(\gamma)$ [18], where $\operatorname{ample}(\gamma) \subseteq \operatorname{enabled}(\gamma)$

We now proceed by investigating how to select a subset ample $(\gamma)$ of enabled $(\gamma)$. For Algorithm 1 to be correct when replacing enabled $(\gamma)$ with $\operatorname{ample}(\gamma)$, the selection of a subset of enabled $(\gamma)$ is guided by the following rules.

B0 The set $\operatorname{ample}(\gamma)$ is empty if and only if enabled $(\gamma)$ is empty.

B1 Each of the operations in ample $(\gamma)$ is contributory to every finite sequence of operations $\rho$ enabled from $\gamma$ that does not contain an operation from ample $(\gamma)$.

It can be shown that selecting a set $\operatorname{ample}(\gamma)$ satisfying conditions $\mathbf{B O}$ and $\mathbf{B 1}$ is not guaranteed to preserve reachability of the initial state. The reason is that because of independence between operations, we may reach a local state in one process before doing so in a second process; we may then continue going backwards in the first process, before reaching the control initial state in the second process and thus we miss the configuration where all processes are in their initial control states. This is a problem inherent in partial order reductions and is not particular for lossy channel systems.

As an example, consider the system in Figure 2. It consists of two processes, executing concurrently. All the transitions (and hence the operations) are local. When trying to search for the initial state $\left(s_{1}, s_{3}\right)$ from the state $\left(s_{2}, s_{3}\right)$, the righthand process has already reached its initial control state. However, according to condition B0, we may select the operation which is the reverse of transition $d$. We then reach the state $\left(s_{2}, s_{4}\right)$. In fact, we may then continue to take the reverse of transition $c$ and reach $\left(s_{2}, s_{3}\right)$ again, terminating the search with the wrong conclusion that the initial state is not reachable.

We have several alternative ways to remedy the problem. One of the simplest ways is to ensure that it is not possible to leave an inital control states once the search has reached it. This can be accomplished by making the system rooted.

Definition 7 A system is rooted if none of its operation is enabled from its initial state. 

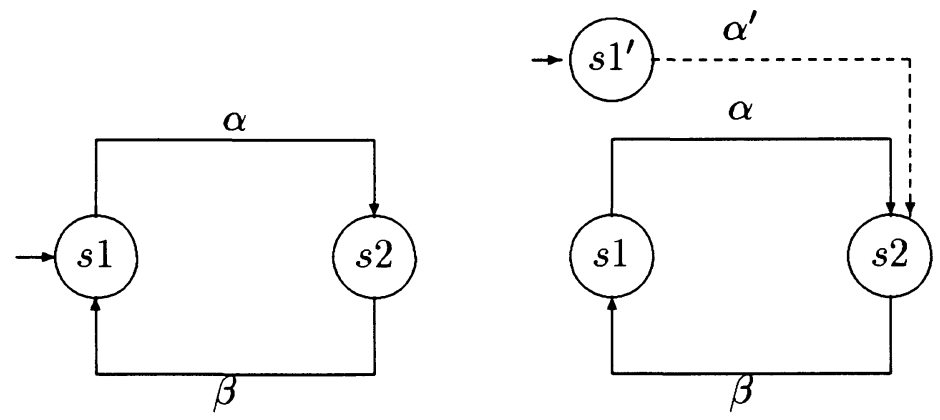

Figure 3 A process transformed to become rooted.

Thus we have our first search strategy:

STRAT1 A backward search in a rooted system (or a system transformed to become rooted), where each ample set satisfies conditions B0 and B1.

Theorem 6 The correctness of the backward reachability problem is preserved under strategy STRAT1.

Proof. It is easy to see that the reduced state space generates only states that can be generated by the full search. Thus, if an initial state is not reachable, the STRAT1 search will terminate (with the same termination argument of the original algorithm [2]) with a negative answer.

For the other direction, assume that an initial state is reachable. Let $\gamma$ be a state of the system, with $\alpha \in \operatorname{enabled}(\gamma) \backslash \operatorname{ample}(\gamma)$, such that there is a path $\alpha \rho$ from $\gamma$ to an initial state $\iota$. Consider first the case where we partition $\alpha \rho$ into a sequence $\sigma \beta \sigma^{\prime}$ such that the sequence $\sigma$ does not contain any operation from ample $(\gamma)$. Then according to condition B1 and Lemma 5, $\beta \sigma \sigma^{\prime}(\gamma) \preceq \sigma \beta \sigma^{\prime}(\gamma)$. Hence $\beta \sigma \sigma^{\prime}$ is a sequence leading to the initial state $\iota$.

Consider now the case that $\alpha \rho$ does not contain any operation from $\operatorname{ample}(\gamma)$. Then, according to condition B0 and B1, ample $(\gamma)$ contains some operation $\beta$ that is contributory to $\alpha \rho$. But then according to Lemma $5, \beta$ is enabled from the initial state $\iota$, contradicting the fact that the system is rooted.

It is easy to see that one can convert any system to a rooted one (if it is not rooted already). The disadvantage of having a rooted system is that it may introduce additional non-determinism. Figure 3 demonstrates a system that is not rooted (on the left) that is converted into a rooted system.

Another strategy, trying to avoid enlarging the state space in order not to miss the initial state, is the following.

Definition 8 A delayed operation is one that can lead out of the initial state. More precisely, $\alpha$ is said to be delayed if $\alpha(\iota)$ is defined. 
In fact, in our case an operation is delayed if and only if it executes from an initial control state of a process. We add the following condition on ample sets:

B2 Either ample $(\gamma)$ contains no delayed operations or ample $(\gamma)=\operatorname{enabled}(\gamma)$.

For example, in the left process in Figure 3, the operation $\beta$ is delayed, since one can take a $\beta$ operation from the initial state. Thus, we have the following search strategy:

STRAT2- A backward search where each ample set satisfies conditions B0, B1, and B2.

Theorem 7 The correctness of the backward reachability problem is preserved under strategy STRAT2.

Proof. Similar to the proof of Theorem 6. The only difference is that in the contradiction case, if $\beta$ is contributory to the sequence $\alpha \rho$ that transforms $\gamma$ into $\iota, \beta$ can still be enabled from $\iota$ (there is no requirement that the system is rooted). However, this means that $\beta$ is a delayed operation. But then according to B2, we must have selected $\operatorname{ample}(\gamma)=\operatorname{enabled}(\gamma)$, contradicting the fact that we did not select $\alpha$.

\section{ALGORITHMS FOR AMPLE SETS}

Having defined conditions for ample sets, we need to provide an algorithm that guarantees selection of a subset of the enabled operations satisfying B0 and B1 and, depending upon the strategy, also B2. Conditions B0 and B2 require only simple checks. However, to guarantee B1, we need to 'predict' what can happen along sequences of operations, starting from the current state. Deciding condition B1 for an arbitrary subset of the enabled transitions can be easily shown to be as difficult as reachability. Instead, some heuristics have to be used.

One simple algorithm is the following.

\section{Algorithm 2 (Simple Algorithm)}

Input: A set of processes $P_{1}, \ldots, P_{n}$ and a state $\gamma$.

Output: A set ample $(\gamma)$ being a subset of enabled $(\gamma)$.

1) For each process $P_{i}$

2) If each operation is either Icl, $\operatorname{rcv} d, m$, or snd $c, m$ with channel $c$ non-empty

3) select ample $(\gamma)$ as the enabled operations of $P_{i}$, exit

4) If there were no process satisfying the above conditions

5) $\quad \operatorname{select} \operatorname{ample}(\gamma)=\operatorname{enabled}(\gamma)$

Lemma 8 The rules B0 and B1 are satisfied when using Algorithm 2 to compute $\operatorname{ample}(\gamma)$. 
Proof sketch. It is trivial to check that BO is satisfied. Observe that B1 is satisfied since all operations belonging to a process are in $\operatorname{ample}(\gamma)$ and that no two processes may receive from the same channel. Hence, it cannot happen that an alternative operation of the same process is disabled but will become enabled by the execution of some snd or rcv event in another process.

To use strategy STRAT2 we alter steps 3 and 6 in Algorithm 2: B2 is applied by selecting the transitions of a certain process only if they also satisfy the condition that they are not delayed.

Theorem 9 Algorithm 1 correctly decides reachability under STRAT1 when using Algorithm 2 to compute ample $(\gamma)$.

Proof. Follows from Theorem 6 and Lemma 8.

\section{EXPERIMENTS}

In this section we consider two examples and show the reductions obtained when using the improved algorithm for reachability.

First we consider the go back $n$ protocol of the well known Sliding Window protocol family. We model the protocol as a lossy channel system with two unbounded and unreliable channels.

In the protocol, $n$ corresponds to the size of the sender window. The receiver window is always of size 1 . We have added a distinguished initial state to make the system rooted.

In Table 1 we compare the performances of Algorithm 1 with and without partial order reduction, for different sizes of the sender window.

\begin{tabular}{crrrrrr}
\hline $\begin{array}{c}\text { Window } \\
\text { Size }\end{array}$ & \multicolumn{2}{c}{ No Partial Order } & \multicolumn{2}{c}{ With Partial Order } & \multicolumn{2}{c}{ Reduction (\%) } \\
& Memory & Time & Memory & Time & Memory & Time \\
\hline 1 & 16152 & 0.02 & 14744 & 0.02 & $9 \%$ & $0 \%$ \\
2 & 92416 & 0.10 & 67024 & 0.08 & $27 \%$ & $20 \%$ \\
3 & 431096 & 0.42 & 297016 & 0.34 & $31 \%$ & $19 \%$ \\
4 & 1446040 & 1.67 & 968184 & 1.26 & $33 \%$ & $25 \%$ \\
5 & 4022080 & 4.94 & 2718224 & 4.24 & $32 \%$ & $14 \%$ \\
6 & 9656504 & 13.34 & 6435560 & 11.22 & $33 \%$ & $16 \%$ \\
7 & 21013480 & 32.71 & 13840888 & 29.81 & $34 \%$ & $9 \%$ \\
\hline
\end{tabular}

Table 1 Verification of Go Back n

In the second example, we consider a token ring to implement mutual exclusion among a number of processes. A number of processes communicate through lossy channels. In Table 2 we compare the performances of Algorithm 1 with and without 
partial order reduction, for different numbers of processes in the ring. The high reduction achieved in this example compared to the first example, is due to the fact that here we have more parallel processes instead of only two processes in the case of the "go back $n$ " protocol.

\begin{tabular}{crrrrrr}
\hline \multirow{2}{*}{ Processes } & \multicolumn{2}{c}{ Algorithm 1 } & \multicolumn{2}{c}{ Algorithm 2 } & \multicolumn{2}{c}{ Reduction (\%) } \\
& Memory & Time & Memory & Time & Memory & Time \\
\hline 4 & 66072 & 0.08 & 48264 & 0.04 & $27 \%$ & $50 \%$ \\
5 & 733200 & 4.18 & 238416 & 0.40 & $67 \%$ & $90 \%$ \\
6 & 5154440 & 161.16 & 1201856 & 5.63 & $77 \%$ & $97 \%$ \\
7 & 34531204 & 6913.97 & 6555268 & 222.07 & $81 \%$ & $97 \%$ \\
\hline
\end{tabular}

Table 2 Verification of the Mutex Protocol

\section{CONCLUSIONS AND FUTURE WORK}

We have considered the application of partial order reduction techniques for lossy channel systems.

In contrast to most existing partial order methods, our approach is applied to a backward reachability algorithm. The choice of transitions selected during the analysis is guided by a preorder which we call the better than relation. This is a weaker relation than the independence relation used in traditional partial order methods, and leads consequently to a more effective analysis. The idea of using the "better than" relation is general as it is applicable to all systems which have "monotone" behaviour, e.g. Petri nets, real-time automata, relational automata, etc. As part of our future research, we shall try to extend our theory to these classes of systems. We also intend to carry out experiments to study the performance of our algorithms on more advanced examples.

\section{REFERENCES}

[1] Parosh Aziz Abdulla and Bengt Jonsson. Verifying programs with unreliable channels. In Proc. $8^{\text {th }}$ IEEE Int. Symp. on Logic in Computer Science, pages 160-170, 1993.

[2] Parosh Aziz Abdulla and Bengt Jonsson. Verifying programs with unreliable channels. Information and Computation, 127(2):91-101, 1996.

[3] Parosh Aziz Abdulla, Karlis Čerāns, Bengt Jonsson, and Tsay Yih-Kuen. General decidability theorems for infinite-state systems. In Proc. $11^{\text {th }}$ IEEE Int. Symp. on Logic in Computer Science, pages 313-321, 1996.

[4] K. Bartlett, R. Scantlebury, and P. Wilkinson. A note on reliable full-duplex transmissions over half duplex lines. Communications of the ACM, 2(5):260-261, 1969.

[5] G. V. Bochmann. Finite state description of communicating protocols. Computer Networks, 2:361-371, 1978. 
[6] D. Brand and P. Zafiropulo. On communicating finite-state machines. Journal of the $A C M, 2(5): 323-342$, April 1983.

[7] J.R. Burch, E.M. Clarke, K.L. McMillan, D.L. Dill, and L.J. Hwang. Symbolic model checking: $10^{20}$ states and beyond. In Proc. ${ }^{\text {th }}$ IEEE Int. Symp. on Logic in Computer Science, 1990.

[8] S. Christensen, Y. Hirshfeld, and F. Moller. Bisimulation equivalence is decidable for basic parallel processes. In Proc. CONCUR' 93, Theories of Concurrency:Unification and Extension, pages 143-157, 1993.

[9] E.M. Clarke, E.A. Emerson, and A.P. Sistla. Automatic verification of finite-state concurrent systems using temporal logic specification. ACM Trans. on Programming Languages and Systems, 8(2):244-263, April 1986.

[10] A. Finkel. Reduction and covering of infinite reachability trees. Information and Computation, (89):144-179, 1990.

[11] S. M. German and A. P. Sistla. Reasoning about systems with many processes. Journal of the ACM, 39(3):675-735, 1992.

[12] P. Godefroid and P. Wolper. Using partial orders to improve automatic verification methods. In Proc. Workshop on Computer Aided Verification, 1990.

[13] G. Higman. Ordering by divisibility in abstract algebras. Proc. London Math. Soc., 2:326-336, 1952.

[14] G.J. Holzmann. Design and Validation of Computer Protocols. Prentice Hall, 1991.

[15] ISO. Data communications - HDLC procedures - elements of procedures. Technical Report ISO 4335, International Standards Organization, Geneva, Switzerland, 1979.

[16] B. Jonsson and J. Parrow. Deciding bisimulation equivalences for a class of non-finitestate programs. Information and Computation, 107(2):272-302, Dec. 1993.

[17] M. Lothaire. Combinatorics on Words, volume 17 of Encyclopedia of Mathematics and its Applications. Addison-Wesley, 1983.

[18] D. Peled. Combining partial order reductions with on-the-fly model checking. Formal Methods in System Design, 8:39-64, 1996.

[19] A. Valmari. On-the-fly verification with stubborn sets. In Courcoubetis, editor, Proc. $5^{\text {th }}$ Int. Conf. on Computer Aided Verification, number 697 in Lecture Notes in Computer Science, pages 59-70, 1993.

[20] M. Y. Vardi and P. Wolper. An automata-theoretic approach to automatic program verification. In Proc. $1^{\text {st }}$ IEEE Int. Symp. on Logic in Computer Science, pages 332-344, June 1986. 